International Journal of Wireless \& Mobile Networks (IJWMN) Vol. 4, No. 4, August 2012

\title{
Performance ANalysis of DSDV and DSR UNDER VARIABLE NODE SPEED IN HYBRID SCENARIO
}

\author{
Koushik Majumder ${ }^{1}$, Sudhabindu Ray $^{2}$ and Subir Kumar Sarkar ${ }^{2}$ \\ ${ }^{1}$ Department of Computer Science \& Engineering, West Bengal University of \\ Technology, Kolkata, INDIA \\ koushikeieee.org \\ ${ }^{2}$ Department of Electronics and Telecommunication Engineering, Jadavpur University, \\ Kolkata, INDIA
}

\begin{abstract}
The mobile ad hoc networks have gained immense popularity in the current decade due their less costly and rapid deployability, inherent support for mobility and the potential to provide ad hoc connectivity to devices. Routing in mobile ad hoc network is considered as a challenging task due to the drastic and unpredictable changes in the network topology resulting from the random and frequent movement of the nodes and due to the absence of any centralized control. Routing becomes even more complex in hybrid networking scenario where the MANET is combined with the fixed network for covering wider network area with less fixed infrastructure. Although, several routing protocols have been developed and tested under various network environments, but, the simulations of such routing protocols have not taken into account the hybrid networking environments. In this work we have carried out a systematic simulation based performance study and analysis of the two prominent routing protocols: Destination Sequenced Distance Vector Routing (DSDV) and Dynamic Source Routing (DSR) protocols in the hy-brid networking environment under varying node speed. We have analyzed the performance differentials on the basis of three metrics - packet delivery fraction, average end-to-end delay and normalized routing load using NS2 based simulation.
\end{abstract}

\section{KEYWORDS}

Mobile ad hoc network, hybrid network scenario, varying node speed, performance analysis, packet delivery fraction, average end-to-end delay, normalized routing load.

\section{INTRODUCTION}

The mobile ad hoc networks(MANET) [1-14] have received increased attention of the research community in the current decade due their self organizing, self controlled and distributed nature of operations which separate them from the fixed networks. The main advantage of these networks is that their non-reliance on any established infrastructure or centralized server. These networks are autonomous where a number of mobile nodes equipped with wireless interfaces communicate with each other either directly or through other nodes. These networks are especially useful in emergency scenarios where there is no fixed infrastructure or the previous infrastructure is totally destroyed and it is not possible to set up a new infrastructure quickly. The communication in MNAET is multi-hop and each node has to play the role of both the host as well as the router. But due to the limited transmission range of the MANET nodes, the total area of coverage is often limited. Also due to the lack of connectivity to the fixed network, the users in the MANET work as an isolated group. However, many applications require connection to the external network such as Internet or LAN to provide the users with external resources. 


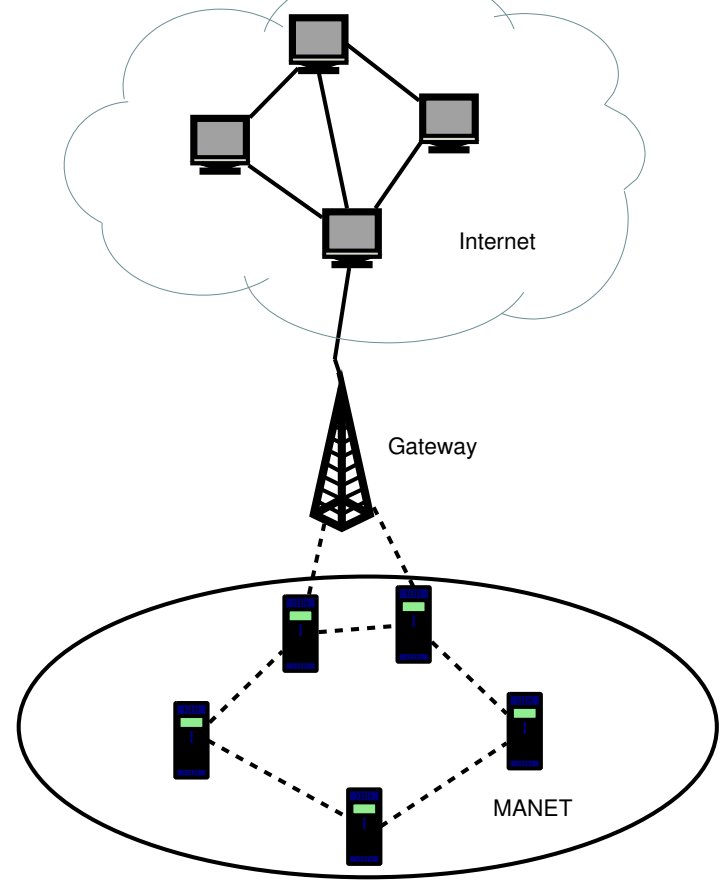

Figure 1. Hybrid Network

On the other hand the growth of Internet has been tremendous in the current decade and with its reducing cost of use, it has occupied a huge part of the lives of the common people. For example people present in any part of the world and connected to the Internet can communicate between them in almost no time using email, online audio and video chat. This plays an important role in the field of academics and research. No longer are the students restricted by their physical presence. For example students in distant areas can participate in the online classroom facilities provided by the top universities, they can download the study materials, raise questions and discuss their problems online. The researchers and scientists in different parts of the world can collaborate and work in groups and exchange their ideas instantaneously. Their distributed geographic presence no longer constrains the scope and rapid growth of research. On the other hand with the huge influx of mobile phones, laptops and personal digital assistants along with their reduced cost, mobility has become an indispensable part of our daily lives. These devices are highly portable and can be carried anytime anywhere. With the increasing use of these devices there is a growing demand for the connectivity to the Internet while we are on the move.

In order to access the global services and applications of the Internet and for widening the coverage area of the MANET, sometimes a hybrid network can be formed by combining the ad hoc network with the wired network. By using this combination we can cover a larger area with less fixed infrastructure, less number of fixed antennas and base station and can reduce the overall power consumption. Due to the hybrid nature of these networks, routing is considered a challenging task. Several routing protocols have been proposed and tested under various traffic conditions. However, the simulations of such routing protocols have not taken into account the hybrid network scenario. In this work we have carried out a systematic performance study of the two prominent routing protocols: Destination Sequenced Distance Vector Routing (DSDV) and Dynamic Source Routing (DSR) protocols in the hybrid networking environment under different node speed. 
International Journal of Wireless \& Mobile Networks (IJWMN) Vol. 4, No. 4, August 2012

The rest of the paper is organized as follows. Section 2 describes the related work. A brief introduction of Dynamic Source Routing (DSR) and Destination Sequenced Distance Vector Routing (DSDV) protocols is given in Section 3. Section 4 and section 5 details the simulation model and the key performance metrics respectively. The simulation results are presented and analyzed in section 6. Finally the conclusion has been summarized in the section 7 . The last section gives the references.

\section{RELATED WORK}

Several simulation based experiments have been made to compare the performance of the routing protocols for mobile ad hoc network.

Das et al. [15] made performance comparison of routing protocols for MANET based on the number of conversations per mobile node for a given traffic and mobility model. Small networks consisting of 30 nodes and medium networks consisting of 60 nodes were used. Simulation was done using the Maryland Routing Simulator (MARS).

Performance comparison results of two on demand routing protocols - AODV and DSR is presented in the work of Das, Perkins and Royer [16]. They used NS2 based simulation. CBR sources were used with packet size of 512 bytes. Two different simulation set ups were used. One with 50 nodes and $1500 \mathrm{~m} \times 300 \mathrm{~m}$ simulation area and the other with 100 nodes and $2200 \mathrm{~m}$ $\mathrm{x} 600 \mathrm{~m}$ simulation area. The performance metrics studied were: packet delivery fraction, average end-to-end delay and normalized routing load.

Johansson, Larssson, Hedman and Mielczarek [17] in their work incorporated new mobility models. A new mobility metric was introduced to characterize these models. Using this metric, mobility was measured in terms of relative speeds of the nodes instead of absolute speeds and pause times. The network consisted of 50 nodes. There were 15 sources and the data packets transmitted were of 64 bytes. Performance analysis was made in terms of throughput, delay and routing load.

Park and Corson [18] made a performance comparison between TORA and an "idealized' link state routing protocol. Many simplifications were made in the simulation environment. For example, in the simulation scenario packets were transmitted at the rate of only 4, 1.5, or 0.6 packets per minute per node for avoiding congestion. Total duration of the simulation run was 2 hours. The network was connected in a "honeycomb" pattern. The node density was kept constant artificially. The notion of true node mobility was missing. Every node was connected to a fixed set of neighboring nodes through separate links. Each link switched between active and inactive states irrespective of other links. Immediate feedback was available when a link went up or down which is not the case in reality.

These works, however, do not take into consideration the influence of hybrid network scenario over the performance of the routing protocols. In this work we have studied the effect of varying node speed on the performance of two prominent routing protocols for mobile ad hoc network Destination Sequenced Distance Vector Routing (DSDV) and Dynamic Source Routing (DSR) protocol in the hybrid networking environment.

\section{DESCRIPTION OF ROUTING PROTOCOLS}

\subsection{Dynamic Source Routing (DSR)}

The Dynamic Source Routing Protocol (DSR) is a reactive routing protocol. The main feature of DSR is the use of source routing technique. In this technique the source node knows the complete hop-by-hop route towards the destination node. The source node lists this entire 
International Journal of Wireless \& Mobile Networks (IJWMN) Vol. 4, No. 4, August 2012

sequence in the packet's header. If a node wants to send a packet to a destination, the route to which is unknown, in that case a dynamic route discovery process is initiated to discover the route. DSR consists of the Route Discovery and Route Maintenance phase, through which it discovers and maintains source routes to arbitrary destinations in the network.

\subsubsection{Route Discovery}

If a node $\mathrm{A}$ wants to send a packet to a destination node $\mathrm{B}$, it searches its Route Cache. If the Route Cache contains a valid route, node $\mathrm{A}$ inserts this route into the header of the packet and sends the data packet to the destination B. In case when no route is found in the Route Cache, a Route Discovery is initiated.

Node A initiates the Route Discovery by broadcasting a ROUTE REQUEST message. All nodes within the transmission range receive this message. The nodes which are not in the route, add their address to the route record in the packet and forward the packet when received for the first time. They check the request id and source node id to avoid multiple retransmissions. The destination node B sends a ROUTE REPLY when it receives a ROUTE REQUEST. If the link is bidirectional, the ROUTE REPLY propagates through the reverse route of the ROUTE REQUEST. If the link is unidirectional, in that case B checks its own Route Cache for a route to A and uses it to send the ROUTE REPLY to the source A. If no route is found, B will start its own Route Discovery. In order to avoid infinite numbers of Route Discoveries it piggybacks the original ROUTE REQUEST message to its own. The route information carried back by the ROUTE REPLY message is cached at the source for future use. In addition to the destination node, other intermediate nodes can also send replies to a ROUTE REQUEST using cached routes to the destination.

\subsubsection{Route Maintenance}

The node which sends a packet using a source route is responsible for acknowledging the receipt of the packet by the next node. A packet is retransmitted until a receipt is received or the maximum number of retransmissions is exceeded. If no confirmation is received, the node transmits a ROUTE ERROR message to the original sender indicating a broken link. The ROUTE ERROR packet causes the intermediate nodes to remove the routes containing the broken link from their route caches. Ultimately the sender will remove this link from its cache and look for another source route to the destination in its cache. If the route cache contains another source route, the node sends the packet using this route. Otherwise, it needs to initialize a new route discovery process. DSR makes very effective use of source routing and route caching. In order to improve performance any forwarding node caches the source route contained in a packet forwarded by it for possible future use.

\subsection{Destination Sequenced Distance Vector Routing (DSDV)}

The Destination Sequenced Distance Vector Routing (DSDV) is a proactive or table driven routing protocol designed for MANET. It was developed by $\mathrm{C}$. Perkins and P. Bhagwat in 19994. This scheme is based on the classical Bellman-Ford distance vector algorithm with certain modifications to make it suitable for the ad hoc environment and to solve the problem of routing loop and count-to-infinity. In DSDV every node maintains a routing table which contains the list of all possible destinations within the network and the number of hops to reach each possible destination. Each distance entry is marked by a sequence number usually originated by the destination node. This sequence numbering scheme is used to counter the count-to infinity problem and to distinguish the stale routes from the fresh ones thus avoiding the formation of loops.

In order to maintain up-to-date routing information about the frequently changing topology of the network the nodes need periodic exchanges of routing tables with their neighbours. But this will create a huge overhead of control packets in an already bandwidth constrained network. To reduce this huge overhead of control traffic the routing updates are generally classified into two 
International Journal of Wireless \& Mobile Networks (IJWMN) Vol. 4, No. 4, August 2012

types - full dump and incremental update. In case of full dumps, nodes need to exchange complete routing tables with their neighbours. Full dumps are needed to maintain consistent routing information when the network topology changes completely and very fast due to frequent movement of nodes. But this may result in a large number of routing packet exchanges between the nodes. On the other hand incremental updates contain only those entries that have been updated since the last full dumps. Incremental updates are much smaller in size than the full dumps and they should fit in a single Network Protocol Data Unit (NPDU). When the network is relatively stable, incremental updates are used to rapidly propagate the routing information regarding the small changes in network topology. This saves a lot of network traffic. In addition to the periodic updates DSDV uses triggered updates, when significant new information is available about the topological change. Thus the update is both time-driven as well as event-driven.

Table updates are initiated by the destination nodes and they generate the sequence numbers. Every node periodically transmits their routing updates to their immediate neighbours with monotonically increasing sequence numbers. After receiving a new route update, every node compares it with its existing entry. Routes with smaller sequence numbers are simply discarded and the one with the recent sequence number is selected. In case when the new route is having the same sequence number as the existing route, the one with the smaller hop count is selected. If the new route is chosen, its hop-count is incremented by one, as the packets will require one more hop to reach the destination. This change in the routing information is then immediately communicated to the neighbours.

When a node $S$ finds that its route to destination $\mathrm{D}$ is broken, it advertises its link to destination $\mathrm{D}$ with an infinite hop-count and a sequence number that is one greater than the sequence number of the broken route. This is the only case when the sequence number is not assigned by the destination node. Sequence numbers defined by the originating nodes are even numbers, whereas the sequence numbers indicating the broken links are odd numbers. After having this infinite hop-count entry, when a node, later receives a finite hop-count entry with newer sequence number, it immediately broadcasts its new routing update. The broken links are thus quickly replaced by the real routes.

\section{SimUlation MODEL}

We have done our simulation based on ns-2.34 [19-22]. NS is a discrete event simulator. It was developed by the University of California at Berkeley and the VINT project [19]. Our main goal was to measure the performance of the protocols under a range of varying network conditions. We have used the Distributed Coordination Function (DCF) of IEEE 802.11[23] for wireless LANs as the MAC layer protocol. Data packets were transmitted using an unslotted carrier sense multiple access (CSMA) technique with collision avoidance (CSMA/CA) [23].

The protocols have a send buffer of 64 packets. In order to prevent indefinite waiting for these data packets, the packets are dropped from the buffers when the waiting time exceeds 40 seconds. The interface queue has the capacity to hold 80 packets and it is maintained as a priority queue. The interface queue holds both the data and control traffic sent by the routing layer until they are transmitted by the MAC layer. The control packets get higher priority than the data packets.

\subsection{Mobility Model}

Inclusion of a mobility model is necessary in order to evaluate the performance of a protocol for ad hoc network in a simulated environment. Here in our work we have used the random waypoint model. This model is a simple and common mobility model and is widely used for the performance evaluation of MANET protocols in simulated environment. This particular mobility model has pause time between changes in direction and/or speed. The mobile nodes are initially distributed over the entire simulation area. In order to ensure randomness in the initial 
International Journal of Wireless \& Mobile Networks (IJWMN) Vol. 4, No. 4, August 2012

distribution data gathering has to start after a certain simulation time. A mobile node starts simulation by waiting at one location for a specified pause time. After this time is over, it randomly selects the next destination in the simulation area. It also chooses a random speed uniformly distributed between a maximum and minimum speed and travels with a speed $v$ whose value is uniformly chosen in the interval $\left(0, v_{\max }\right)$. Then the mobile node moves towards its selected destination at the selected speed. After reaching its destination, the mobile node again waits for the specified pause time before choosing a new way point and speed.

\subsection{Movement Model}

In the simulation environment the nodes move according to our selected random waypoint mobility model.We have generated the movement scenario files using the setdest program which comes with the NS-2 distribution. The total duration of our each simulation run is 900 seconds. We have varied our simulation with movement patterns for six different node speed: $5 \mathrm{~m} / \mathrm{s}, 10 \mathrm{~m} / \mathrm{s}, 15 \mathrm{~m} / \mathrm{s}, 20 \mathrm{~m} / \mathrm{s}, 25 \mathrm{~m} / \mathrm{s}, 30 \mathrm{~m} / \mathrm{s}$. We have performed our experiment with two different numbers of source nodes: 30 source nodes and 40 source nodes. As slight changes in the movement pattern will have significant effect on the protocol performance, we have generated scenario files with 60 different movement patterns, 10 for each value of node speed. In order to compare the performance of the protocols based on the identical scenario both the protocols were run with these 60 different movement patterns.

\subsection{Communication Model}

In our simulation environment the MANET nodes use constant bit rate (CBR) traffic sources. We have used the cbrgen traffic-scenario generator tool available in NS2 to generate the CBR traffic connections between the nodes. Data packets transmitted are of 512 bytes. Data packets are sent at the rate of 5 packets/second.We have used two different communication patterns corresponding to 30 and 40 sources. The complete list of simulation parameters is shown in Table 1.

Table 1. Simulation Parameters.

\begin{tabular}{|l|l|}
\hline Parameter & Value \\
\hline Protocols & DSDV, DSR \\
\hline Number of mobile nodes & 70 \\
\hline Number of fixed nodes & 10 \\
\hline Number of sources & 30,40 \\
\hline Transmission range & $250 \mathrm{~m}$ \\
\hline Simulation time & $900 \mathrm{~s}$ \\
\hline Topology size & $900 \mathrm{~m}$ X $600 \mathrm{~m}$ \\
\hline Source type & Constant bit rate \\
\hline Packet rate & 5 packets $/ \mathrm{sec}$ \\
\hline Packet size & 512 bytes \\
\hline Pause time & 100 seconds \\
\hline Node speed & $5 \mathrm{~m} / \mathrm{s}, 10 \mathrm{~m} / \mathrm{s}, 15 \mathrm{~m} / \mathrm{s}, 20 \mathrm{~m} / \mathrm{s}, 25 \mathrm{~m} / \mathrm{s}$, \\
& $30 \mathrm{~m} / \mathrm{s}$ \\
\hline Mobility model & Random way point \\
\hline
\end{tabular}


International Journal of Wireless \& Mobile Networks (IJWMN) Vol. 4, No. 4, August 2012

\subsection{Hybrid Scenario}

We have used a rectangular simulation area of $900 \mathrm{~m}$ x $600 \mathrm{~m}$. The choice of rectangular area instead of square area was made in order to ensure longer routes between nodes. In our simulation we have used two ray ground propagation model. Our mixed scenario consists of a wireless and a wired domain. The simulation was performed with 70 wireless nodes and 10 wired nodes. For our hybrid networking environment we have a base station located at the centre $(450,300)$ of the simulation area. The base station acts as a gateway between the wireless and wired domains. For our mixed simulation scenario we have turned on hierarchical routing in order to route packets between the wired and the wireless domains. The domains and clusters are defined by using the hierarchical topology structure. As the base station nodes act as gateways between the wired and wireless domains, they need to have their wired routing on. In the simulation setup we have done this by setting the node-config option -wiredRouting on. After the configuration of the base station, the wireless nodes are reconfigured by turning their wiredRouting off.

\section{Performance Metrics}

We have primarily selected the following three performance metrics in order to study the performance comparison of DSDV and DSR.

Packet delivery fraction: This is defined as the ratio between the number of delivered packets and those generated by the constant bit rate (CBR) traffic sources.

Average end-to-end delay: This metric includes all possible delays caused by buffering at the time of the route discovery, queuing delay due to waiting at the interface queue, retransmission delays at MAC, propagation and transfer times. This is basically defined as the ratio between the summation of the time difference between the packet received time and the packet sent time and the summation of data packets received by all nodes.

Normalized routing load: This is defined as the number of routing packets transmitted per data packet delivered at the destination. Each hop-wise transmission of a routing packet is counted as one transmission.

\subsection{Packet Delivery Fraction (PDF) Comparison}

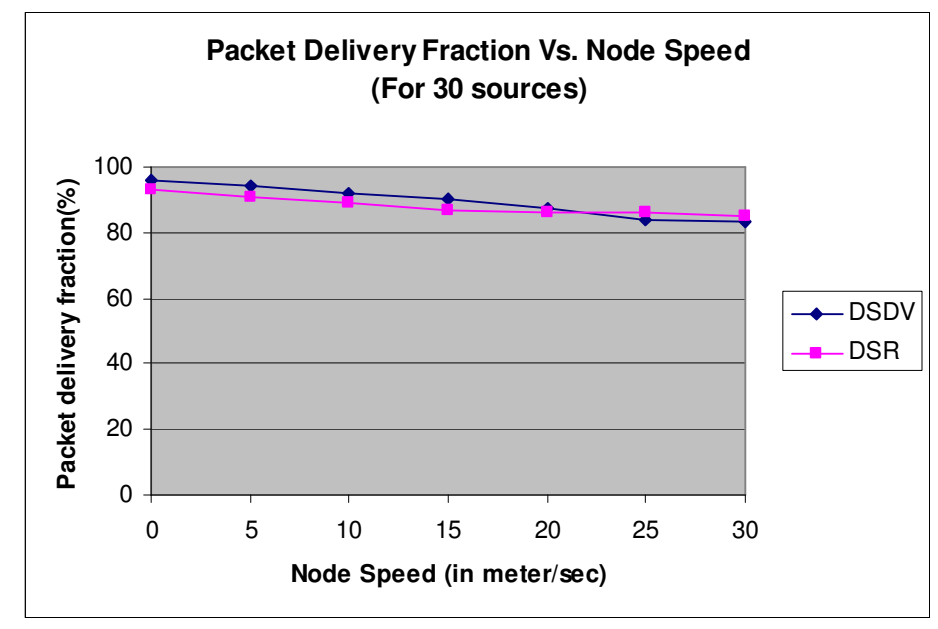

Figure 2. Packet Delivery Fraction Vs. Node Speed for 30 sources 


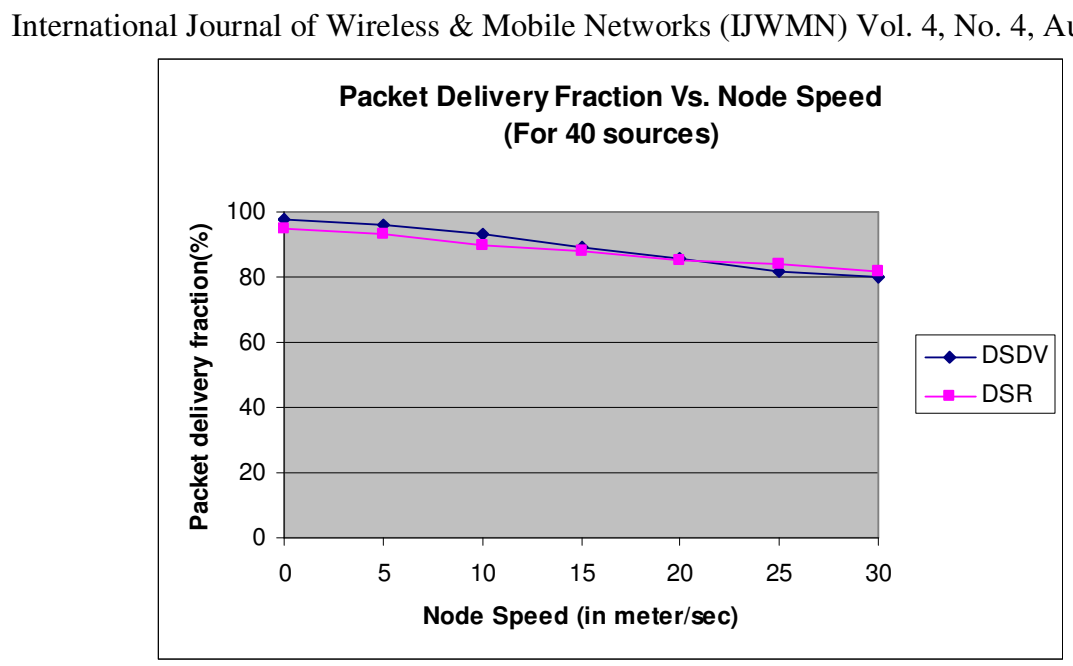

Figure 3. Packet Delivery Fraction Vs. Node Speed for 40 sources

From Fig. 2 and Fig. 3 we observe the difference in the packet delivery performances of DSDV and DSR from our simulation experiments. We have measured the packet delivery fraction of these two protocols by varying the node speed with respect to 30 and 40 numbers of sources. From the graphs we see that DSDV shows better packet delivery performance than DSR at lower node speed. This happens due to the fact that, at lower node speed, the network remains relatively stable and once a route is established, it continues to be available for a longer period of time. Due to the proactive nature of DSDV, routing information exchanges take place regularly between the nodes and each node maintains routing information to every destination all the time. Consequently, most of the packets can be delivered smoothly without having to wait for the path setup time. This results in better packet delivery performance of DSDV. On the contrary, DSR, being a source routing protocol, a significant time is required for initial path setup. During this time, no packets can be delivered to the destination due to unavailability of routes. This results in lower packet delivery fraction of DSR in comparison to DSDV.

With higher node speed, the network topology becomes highly dynamic and link breaks become more frequent. The unavailability of routes causes the nodes to show deterioration in the packet delivery performance for both DSDV and DSR. The periodic nature of operation of DSDV makes it less adaptive to these frequent changes. It requires greater number of full dumps to be exchanged between the nodes in order to maintain up-to-date routing information at the nodes. This huge volume of control traffic occupies a significant part of the channel bandwidth and lesser channel capacity remains available for the data traffic which results in reduced packet delivery fraction of DSDV at higher node speed.

DSR on the contrary, is more adaptive to the frequently changing scenario due to its on-demand nature of functioning. DSR maintains multiple routes in the cache. Thus, even if a link is broken due to higher node speed, alternative routes can be obtained from the cache. This reduces the number of dropped packets and results in better packet delivery performance of DSR.

It can also be noticed from the figures that as the number of sources is increased, initially when the network topology remains relatively stable at lower node speed, the packet delivery fraction also gets increased. This happens due to the fact that with lesser number of sources, the channel capacity is not fully utilized. Hence, increasing the number of sources also improves the packet delivery ratio. However, when the node speed is increased more along with greater number of sources, finding the route requires greater amount of routing traffic. This leads to reduced 
International Journal of Wireless \& Mobile Networks (IJWMN) Vol. 4, No. 4, August 2012 availability of the channel bandwidth for data transmission and more congestion which ultimately reduces the packet delivery ratio.

\subsection{Average End-to-End Delay Comparison}

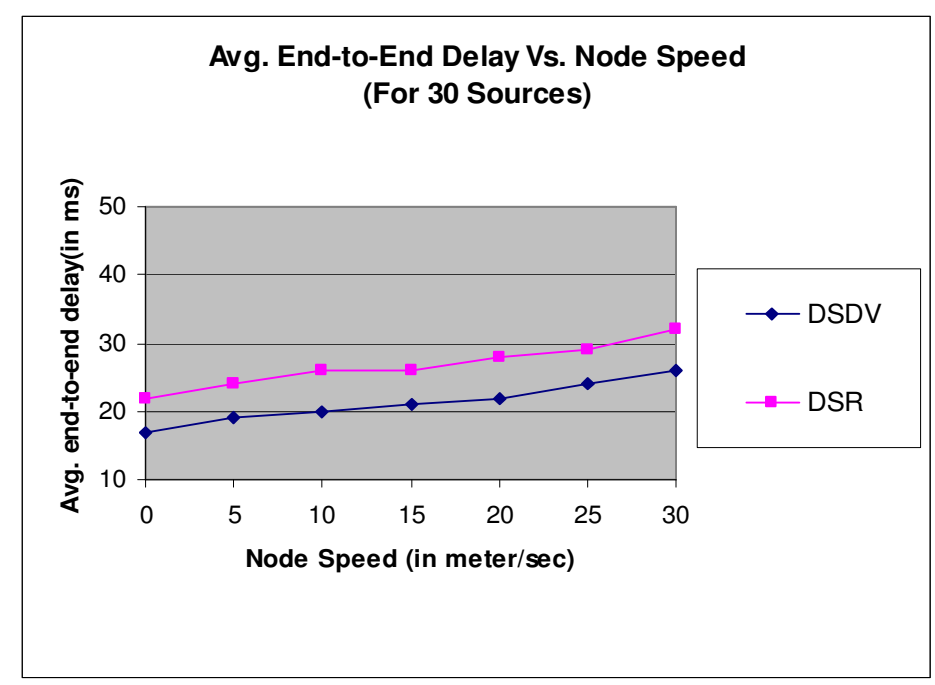

Figure 4. Average End to End Delay Vs. Node Speed for 30 sources

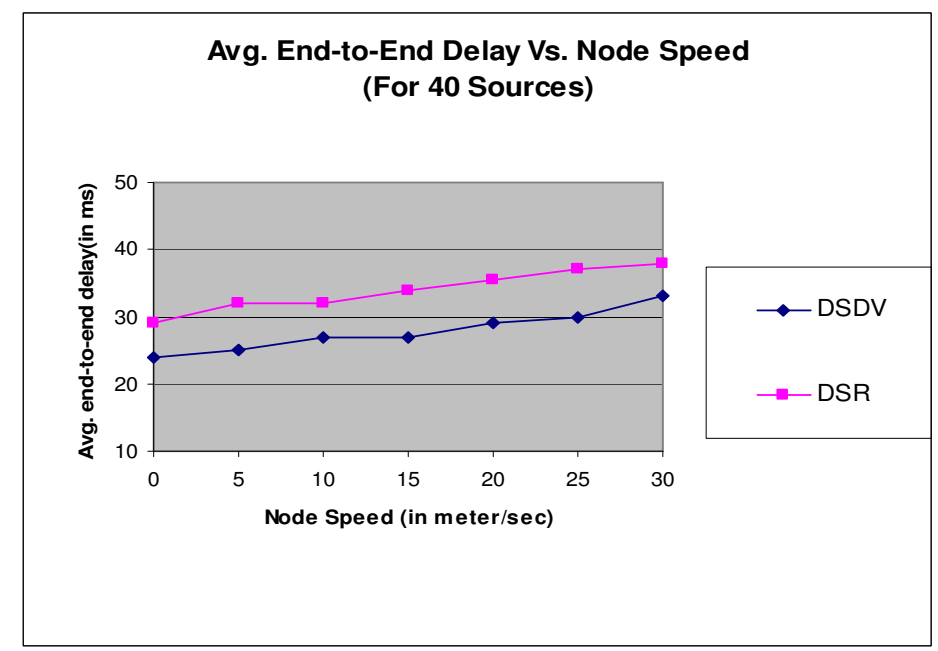

Figure 5. Average End to End Delay Vs. Node Speed for 40 sources

From Fig. 4 and Fig. 5 we can observe the fact that DSDV has less average end to end delay in comparison to DSR. DSDV is a proactive routing protocol. In DSDV, nodes periodically exchange routing tables between them in order to maintain up-to-date routing information to all destinations. Due to this regular route optimization, nodes have access to fresher and shorter routes to the destinations all the time. Hence, whenever a source node wants to send a packet to a destination node, with the already available routing information it can do so without wasting any time for path setup. This instant availability of fresher and shorter routes thus results in less average end-to-end delay in the delivery of data packets in case of DSDV. 
International Journal of Wireless \& Mobile Networks (IJWMN) Vol. 4, No. 4, August 2012

DSR, on the contrary, is a reactive source routing protocol and routing information exchanges do not take place regularly. Instead, if a node in DSR wants to send a packet to a destination node, it has to first find the route to the destination in an on demand fashion. This route discovery latency is a part of the total delay. DSR being a source routing protocol, the initial path set up time is significantly higher as during the route discovery process, every intermediate node needs to extract the information before forwarding the data packet. Moreover in DSR, the source needs to wait for all the replies sent against every request reaching the destination. This increases the delay.

From the figures it is evident that the average end-to-end delay becomes more with higher node speed and greater number of sources for both the protocols. Frequent changes in the network topology due to increasing node speed results in greater number of link breaks. This together with the greater number of sources requires DSR to invoke the route discovery process more frequently in order to find new routes. The frequent invocation of the route discovery creates huge amount of control traffic. The data traffic to be delivered also becomes more with greater number of sources. This results in more collisions, further retransmissions and higher congestion in the network. Consequently, the route discovery latency increases due to the constrained channel. This in turn increases the average end-to-end delay. In addition to that, due to the higher priority of the control packets, the data packets need to spend more time in the queue waiting for the huge volume of control packets to be delivered. This also increases the end-to-end delay in delivering the data packets. In case of DSDV, due to higher speed of the nodes and frequent link breaks, routes become unavailable and nodes need to wait till the next routing information exchanges for new routes. Thus the delay increases depending upon the duration of the interval between the successive routing information exchanges.

\subsection{Normalized Routing Load Comparison}

From Fig. 6 and Fig. 7 we note that initially at lower node speed, DSR has greater normalized routing load. This is attributed to the fact that DSR being a source routing protocol, with every packet the entire routing information is embedded. In addition to that, in response to a route discovery, replies come from many intermediate nodes. This increases the total control traffic. In case of DSDV, initially, at lower node speed, the network topology remains relatively stable. Hence, nodes need to exchange only incremental dumps rather than full dumps. This results in lesser overhead of DSDV.

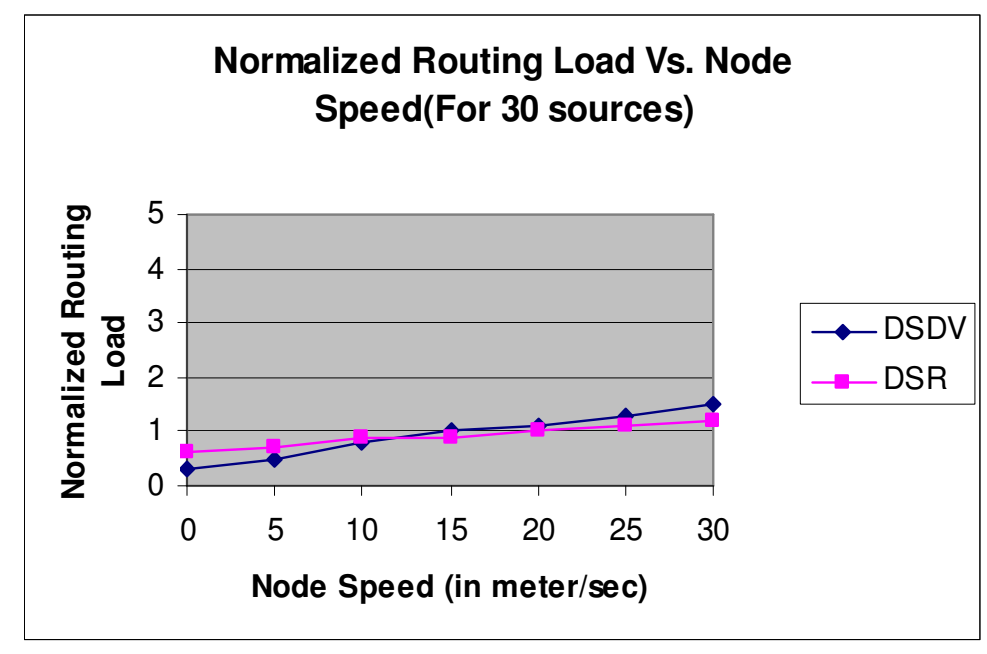

Figure 6. Normalized Routing Load Vs. Node Speed for 30 sources 
International Journal of Wireless \& Mobile Networks (IJWMN) Vol. 4, No. 4, August 2012

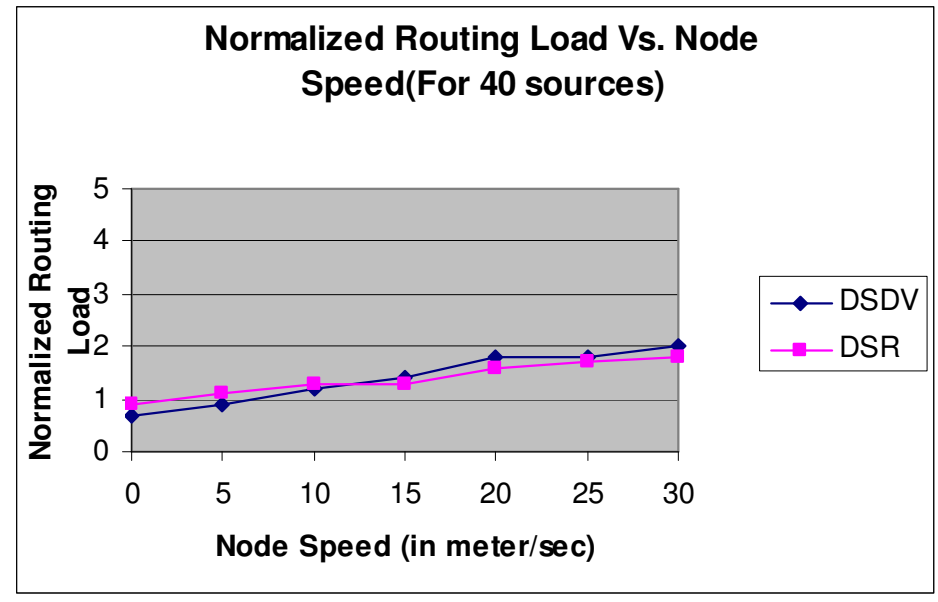

Figure 7. Normalized Routing Load Vs. Node Speed for 40 sources

Both DSDV and DSR suffer from increased normalized routing load with higher node speed and greater number of sources. In case of DSR, with increasing node speed, the route discoveries need to be invoked more often due to increase in the number of broken links. Furthermore, as DSR does not use route optimization until the route is broken and continues using longer and older routes, the chances of link breaks also increase. This further adds to the number of route discoveries which ultimately results in huge control traffic and subsequently higher normalized routing load. Greater number of sources also causes frequent invocation of the route discovery which significantly increases the volume of control overhead. Higher volume of data and control traffic creates congestion in the network. This results in further collisions, more retransmissions and newer route discoveries and further adds up to the already increased control overhead which ultimately results in higher normalized routing load.

With higher node speed, the network topology experiences frequent and high volume of changes. DSDV, due to its proactive nature of operation, is less adaptive to this highly dynamic scenario. Therefore, nodes need to exchange full dumps in order to maintain up-to-date routing information. This causes greater routing overhead for DSDV. In comparison, DSR uses aggressive caching strategy and the hit ratio is quite high. As a consequence, in highly dynamic scenario, even if a link breaks, DSR can resort to an alternate link already available in the cache. Thus the route discovery process can be postponed until all the routes in the cache fail. This reduces the frequency of route discovery, which ultimately results in less routing overhead of DSR.

\section{Conclusions}

In this paper we have carried out a detailed ns2 based simulation to study and analyze the performance differentials of DSDV and DSR in the hybrid scenario under varying node speed with different number of sources. Our work is the first in an attempt to compare these protocols in hybrid networking environment. From the simulation results we see that at lower node speed, DSDV shows better packet delivery performance than DSR mainly due to the instant availability of fresher and newer routes all the time. On the other hand, with higher node speed, DSDV shows more deterioration in the packet delivery performance than DSR mainly due to its less adaptability to the highly dynamic network topology. DSR's better performance is attributed to its ability to maintain multiple routes per destination and its use of aggressive caching strategy. In terms of the average end-to-end delay, DSDV outperforms DSR. The poor performance of DSR in terms of average end-to-end delay is primarily due to its source routing 
International Journal of Wireless \& Mobile Networks (IJWMN) Vol. 4, No. 4, August 2012

nature and its inability to expire the stale routes. Both the approaches suffer form greater average end-to-end delay when we increase the speed of the nodes and the numbers of sources. At higher node speed we observe that DSR shows lower routing load in comparison to DSDV. DSR applies aggressive caching technique and maintains multiple routes to the same destination. Hence, in highly dynamic scenario, even if a link is unavailable due to link break, DSR can resort to an alternate link already available in the cache. This results in reduced frequency of route discovery which ultimately reduces the routing overhead of DSR. On the other hand, at lower node speed, the network topology remains relatively stable. Hence, in DSDV, nodes need to exchange only incremental dumps rather than full dumps. This results in lesser overhead of DSDV. Thus we can conclude that if routing delay is of little concern, then DSR shows better performance at higher mobility in terms of packet delivery fraction and normalized routing load in hybrid networking scenario. Under less stressful scenario, however, DSDV outperforms DSR in terms of all the three metrics.

\section{REFERENCES}

[1] Dow, C. R, (March 2005) A Study of Recent Research Trends and Experimental Guidelines in Mobile Ad-Hoc Networks, In: Proceedings of 19th International Conference on Ad-vanced Information Networking and Applications, IEEE, Vol. 1, pp. 72-77.

[2] Freisleben, B., Jansen, R, (1997) Analysis of Routing Protocols for Ad hoc Networks of Mobile Computers, In: Proceedings of the 15th IASTED International Conference on Applied Informatics, IASTED-ACTA Press, pp. 133-136, Innsbruck, Austria.

[3] Royer, E. M. , Toh, C. K, (April 1999) A Review of Current Routing Protocols for Ad hoc Mobile Wireless Networks, IEEE Personal Communications Magazine, pp. 46-55.

[4] Anastasi, G., Borgia, E., Conti, M., Gregori, E, (2003) IEEE 802.11 Ad-hoc Networks: Protocols, Performance and Open Issues, Ad hoc Networking. IEEE Press Wiley, New York.

[5] Arun Kumar, B. R., Reddy, Lokanatha C., Hiremath, Prakash S, (January-June 2008) A Survey of Mobile Ad hoc Network Routing Protocols, Journal of Intelligent System Research.

[6] Rappaport, T. S, (1996) Wireless Communications, Principles \& Practices. Prentice Hall.

[7] Vaidya, Nitin H, (2004) Mobile Ad Hoc Networks: Routing, MAC and Transport Issues, Tutorial presented at INFOCOM 2004 (IEEE International Conference on Computer Communication), University of Illinois at Urbana-Champaign.

[8] Arun Kumar, B. R., Reddy, Lokanatha C., Hiremath, Prakash S, (2008) Mobile Ad hoc Networks: Issues, Research Trends and Experiments, International Engineering and Technology (IETECH) Journal of Communication Techniques, Vol. 2, No. 2.

[9] Toh, C. K, (2002) Ad-Hoc Mobile Wireless Networks. Prentice Hall.

[10] Tanenbaum, Andrew S, (2002) Computer Networks. Fourth Edition. Prentice Hall.

[11] Corson, S. , Macker, J, (January 1999) Mobile Ad hoc Networking (MANET): Routing Protocol Performance Issues and Evaluation Considerations, IETF MANET Working Group RFC-2501.

[12] Murthy, C. S. R., Manoj, B. S, (2004) Ad Hoc Wireless Networks: Architecture and Protocols, Prentice Hall Communications, Engineering and Emerging Technologies Series, New Jersey.

[13] Comer, D, (2000) Internetworking with TCP/IP, Volume 1, Prentice Hall.

[14] Blum, Jermy I., Eskandarian, Azim , Ho_man, Lance J, (Dec. 2004) Challenges of inter-vehicle Ad hoc Networks, IEEE transactions on Intelligent Transportation Systems. Vol. 5, No. 4.

[15] Das, S. R., Castaeda, R., Yan, J, (2000) Simulation-based Performance Evaluation of Routing Protocols for Mobile Ad hoc Networks, Mobile Networks and Applications, Vol. 5, pp. 179-189. 
International Journal of Wireless \& Mobile Networks (IJWMN) Vol. 4, No. 4, August 2012

[16] Das, Samir R., Perkins, Charles E., Royer, Elizabeth M, (March 2000) Performance Comparison of two On-demand Routing Protocols for Ad hoc Networks, In: Proceedings of the IEEE Conference on Computer Communications (INFOCOM), Tel Aviv, Israel, pp. 3-12.

[17] Johansson, P., Larsson, T., Hedman, N., Mielczarek, B, (August 1999) Routing Protocols for Mobile Ad-hoc Networks - A Comparative Performance Analysis, In: Proceedings of the 5th International Conference on Mobile Computing and Networking (ACM MOBICOM'99).

[18] Park, Vincent D., Corson, M. Scott, (June 1998) A Performance Comparison of TORA and Ideal Link State Routing, In: Proceedings of IEEE Symposium on Computers and Communication '98.

[19] Fall, K., Vardhan, K. Eds, (1999) Ns notes and documentation, available from, http://www.mash.cd.berkeley.edu/ns/.

[20] Network Simulator-2 (NS2), http://www.isi.edu/nsnam/ns

[21] The CMU Monarch Project: The CMU Monarch Projects Wireless and Mobility Extensions to ns, http://www.monarch.cs.cmu.edu (1998).

[22] Altman, E., Jimenez, T, (2003) NS Simulator for Beginners, Lecture notes. Univ. de Los Andes, Merida, Venezuela and ESSI. Sophia-Antipolis, France.

[23] IEEE Computer Society LAN MAN Standards Committee, (1997) Wireless LAN Medium Access Control (MAC) and Physical Layer (PHY) Specifications, IEEE Std 802.11-1997. The Institute of Electrical and Electronics Engineers, New York.

\section{Authors}

Koushik Majumder has received his B.Tech and M.Tech degrees in Computer Science and Engineering and Information Technology in the year 2003 and 2005 respectively from University of Calcutta, Kolkata, India. Before coming to the teaching profession he has worked in reputed international software organizations like Tata Consultancy Services and Cognizant Technology Solutions. He is presently working as an Assistant Professor in the Dept. of Computer Science \& Engineering in West Bengal University of Technology, Kolkata, India He is currently working towards his

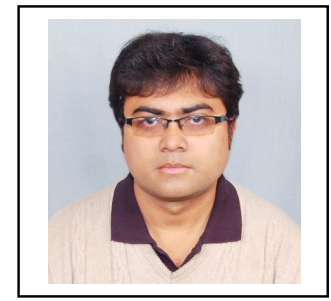
$\mathrm{PhD}$ degree in the Area of Mobile Ad hoc Networks. He has published several papers in International and National level journals and conferences.

Dr. Sudhabindu Ray received his BE (Electronics) and ME (TeleCommunication and Digital Systems and Instrumentation) degrees in 1997 and 1999, respectively from Bengal Engineering and Science University (Bengal Engineering College). He obtained his $\mathrm{PhD}$ degree in the field of communication engineering (Microwave Planar Circuits and Antennas) in 2004 from IIT Bombay. He has worked as a lecturer at Bengal Engineering and Science University (Bengal Engineering College). He is presently working as an Associate Professor in the Department of Electronics and Telecommunication Engineering, Jadavpur University, Kolkata, INDIA. His

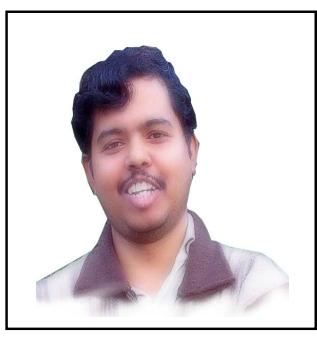
area of Interest are microstrip antenna \&passive circuit, filters, medical electronics and mobile computing. He has published several papers in International and National level journals and conferences. 
International Journal of Wireless \& Mobile Networks (IJWMN) Vol. 4, No. 4, August 2012

Prof Subir Kumar Sarkar received the B. Tech and M. Tech. Degree from the Institute of Radio Physics and Electronics, University of Calcutta in 1981 and 1983, respectively and PhD (Tech) degree in Microelectronics from University of Calcutta. He served Oil and Natural Gas Commission (ONGC) as an Executive Engineer for about 10 years( 1982 to 1992) before coming to teaching profession. He joined as a faculty member in the Dept. of Electronics and Telecommunication Engineering, Bengal Engineering and Science University, Shibpur in April 1992 (from 1992 to 1999). In 1999 he joined in Jadavpur University in the same dept. where he is presently a

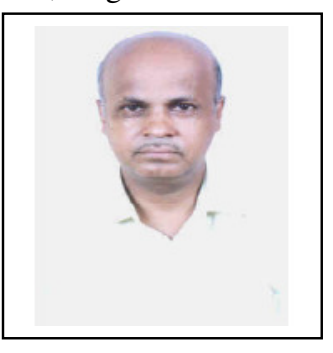
Professor. He has developed several short courses for the needs of the Engineers. He has published three Engineering text books and more than 250 technical research papers in archival journals and peer - reviewed conferences. His most recent research focus is in the areas of simulations of nanodevice models ,transport phenomenon, single electron \& spintronics devices and their applications in VLSI circuits, ad hoc wireless networks, wireless mobile communication and data security. He is a Senior Member, IEEE , Fellow of the Institution of Engineers, and member of Indian Association for the cultivation of Science. 